\title{
Categorization of Bacterial Pathogens Present in Infected Wounds and their Antibiotic Resistance Profile Recovered from Patients Attending Rizgary Hospital-Erbil
}

\author{
Ahmed A. Al-Naqshbandi ${ }^{1}$, Hedy A. Hassan², Mahmoud A. Chawsheen ${ }^{3}$ and Haval H. Abdul Qader ${ }^{4}$ \\ ${ }^{1}$ Department of Laboratory, Rizgary Teaching Hospital, \\ Erbil, Kurdistan Region - F.R. Iraq \\ ${ }^{2}$ Department of Clinical Biochemistry, College of Health Sciences, Hawler Medical University, \\ Erbil, Kurdistan Region - F.R. Iraq \\ ${ }^{3}$ Department of General Sciences, Faculty of Education, Soran University, \\ Erbil, Kurdistan Region - F.R. Iraq \\ ${ }^{4}$ Nanakali Hospital for Blood Diseases and Cancer, \\ Erbil, Kurdistan Region - F.R. Iraq
}

\begin{abstract}
Wound infection with antibiotic-resistant bacteria can extend a patients' debility and increase the expense of treatment in the long term; therefore, careful management of patients with wound infections is necessary to avoid complications. The usage of antimicrobial agent is a major factor in resistance development. This study aims to understand the causes of wound infections, as well as the criteria for diagnosing them for more sensible antibiotic prescribing. Samples from 269 wound patients were collected, and cultured for bacterial growth. Gram stain technique, bacterial identification through VITEK 2 compact system was investigated in this study. Gram-negative bacteria (GNB) accounted for $\mathbf{5 9 . 1 5 \%}$ of the total isolates, whereas pathogenic Gram-positive bacteria (GPB) accounted for $\mathbf{4 0 . 8 5 \%}$ of total isolates. Escherichia coli and Pseudomonas aeruginosa are the dominant pathogenic GNB in wounds, whereas Staphylococcus aureus and Staphylococcus epidermidis are the dominant pathogenic GPB. P. aeruginosa showed $100 \%$ resistance to the majority of antibiotic tested, including Ampicillin, Amoxicillin/Clavulanic Acid, Aztreona, Ceftriaxone, and others. S. aureus and $S$. epidermidis are $100 \%$ resistant to Ampicillin, Ceftriaxone, and Cefotaxime. For more efficient antibiotic prescriptions, the causative microorganisms, and their current susceptibility patterns need to be mandated for testing before prescribing any antibiotics to patients. Prescriptions are frequently based solely on general information about the antibiotic's function, rather than on individual response variation to the pathogen and the antibiotic. Particularly, when the common pathogens in this study show multidrug resistance in wounds.
\end{abstract}

Index Terms-Antibiotic resistance, Healing, Infection, Multi drug resistant, Pathogenic bacteria, Wound.

ARO-The Scientific Journal of Koya University Vol. IX, No.2 (2021), Article ID: ARO.10864, 7 pages DOI: $10.14500 /$ aro. 10864

Received: 16 August 2021; Accepted: 13 November 2021;

\section{;}

Regular research paper: Published: 01 December 2021
Corresponding author's email: mahmoud.hassan@soran.edu.iq Copyright (C) 2021 Ahmed A. Al-Naqshbandi, Hedy A. Hassan, Mahmoud A. Chawsheen and Haval H. Abdul Qader. This is an open access article distributed under the Creative Commons Attribution License.

\section{INTRODUCTION}

Globally, infection with multidrug-resistance bacteria is the main contributing agent to nearly 700,000 deaths. This estimate is expected to rise to up to 10 million deaths by the year 2050 (Reale, et al., 2017). The main route of bacteria entry to the body is through open wounds. Among the four wound cleanliness classifications established by the US Centers for Disease Control and Prevention, clean, clean-contaminated, contaminated, and dirty-infected, the fourth class shows harm to skin, mucous membranes, and organs and can demonstrate microorganism infection and inflammation (Herman and Bordoni, 2021). Wound healing processes involve repair of the damaged tissue with the help of platelets, immune cells, fibroblasts, microvascular cells, and keratinocytes. The healing process is divided into four phases that coinciding with each other; coagulation, inflammation, proliferative (formation of granulation tissue), and formation (remodeling) phases (Chadwick and Ousey, 2019). Chronic non-healing wounds begin as minor damage, including those caused by insect bites, skin scratches, or deep skin penetration. Minor injuries usually heal in a few days or weeks, but in abnormal health conditions such as diabetes, the healing process takes much longer (DemidovaRice, et al., 2012). Chronic wounds have been classified into diabetic ulcers, pressure ulcers, and vascular ulcers. These types of chronic wounds are characterized by a longer inflammatory phase, infections, presence of biofilms, and unresponsiveness to skin healing stimuli (Frykberg and Banks, 2015). Infections with more than 105 live bacteria (or A-hemolytic streptococci) can cause harmful damage to the body. Viable bacteria or their toxins cause an inflammatory response, which can result in abscess, cellulites, and osteomyelitis. In addition to an increase in the production of Matrix metalloproteinases (MMPs) with the aid of the host's immune cells. The MMP breaks down extracellular matrix and growth factors needed for tissue recovery in wounded regions. Bacteria are colonized in wounds form a biofilm. They are often associated with delayed wound healing (Demidova-Rice, et al., 2012). The bacterial capability 
to endure antimicrobial agents has two components; antibiotic tolerance and antibiotic resistance. Tolerance is the bacterial ability to maintain their physical state in the presence of the antibiotic. These biofilms have a high tolerance to antimicrobial agents, but when the biofilm is disrupted the microbes become susceptible to antibiotic treatment (Chadwick and Ousey, 2019; Cooper, et al., 2014). Antibiotic resistance emerges as a result of the response to antibiotic exposure. It has developed naturally over billions of years as a microorganism's successful survival strategy. However, bacteria have evolved antibiotic resistance mechanisms in $<80$ years since their introduction due to the overuse of antibiotics in human and animal health. Antibiotic resistance is one of the most serious risks to human health today. As a result, antimicrobial wound management remains a significant challenge that necessitates new approaches to combat microbes and their biofilms (Bowler, 2018; Daeschlein, 2013).

Gram-positive bacteria (GPB) are typically the first to penetrate the wound space; coagulase-negative staphylococci are the most common group obtained as commensals from the physiological milieu of unbroken skin in the wound's vicinity. Days to weeks later, Gram-negative bacteria (GNB), primarily rods, penetrate the field and compete with surviving species, depending on the patient's specific immunological habitat control. These germs usually come from sanitary barrier failures in everyday hospital hygiene, nutritional supply, and water. Other important microbial players in acute wounds are Pseudomonas aeruginosa and E. coli. Gramnegative rods (Enterobacteriaceae) and $P$. aeruginosa are the most common species found in chronic wound biofilms, followed by Gram-positive cocci such as fecal streptococci (Enterococcus faecalis and Enterococcus faecium) and Staphylococcus aureus (Bowler, 2018; Negut, et al., 2018).

Because of the essential functional and esthetic role of this tissue, the treatment of wounds is a key research domain. When the skin's barrier function is compromised, bacteria may quickly invade the underlying tissues, resulting in life-threatening infections. As a result, successful therapies for such pathological conditions are needed (Bjarnsholt, 2013). This study aims to describe the antibiotic resistance profile for bacterial pathogens present in infected wounds and make an assessment of the most suitable antibiotic with the best impact on wound healing.

\section{Materials AND Methods}

\section{A. Specimen Collection and Transport}

Wound swaps were collected directly from patients attended Rizagary Teaching Hospital in Erbil city for the period between: January, 2014 and December, 2016. After the collection, 269 specimens were transported to laboratories of the microbiology department for analysis.

\section{B. Bacterial Culture and Identification}

After collection specimens were inoculated separately on: Blood, chocolate, and MacConkey agar. The inoculum was first smeared thoroughly over the surface of the solidified medium by sterilized loop. The loop was sterilized again and drawnout from the first site of inoculation into three parallel lines on fresh surfaces of the medium. Series of strokes were made in succession, with the inoculum derived from the most distal part of the immediately preceding strokes at each step. The plates were incubated overnight at $37^{\circ} \mathrm{C}$. Number of colonies was counted and bacterial numbers were calculated per $\mathrm{ml}$ of specimen. For aerobic bacterial growth, colony characteristics and Gram's staining were considered for their identification (Kumar, 2016). Classification of GPB and GNB was performed by following the VITEK $^{\circledR} 2$ compact system (bioMérieux S.A., France) using the following kits: VITEK ${ }^{\circledR} 2$ GN Reference 21341, VITEK $^{\circledR} 2$ GP Reference 21342, and VITEK ${ }^{\circledR} 2$ AST-GN 82 Reference 413439.

\section{Antibiotics}

Antimicrobial sensitivity tests were performed using the VITEK ${ }^{\circledR}$ 2 compact system (bioMérieux S.A., France) with the following kits: VITEK ${ }^{\circledR}$ AST-P580 Reference 22233, and VITEK ${ }^{\circledR}$ ASTST01 Reference 410028. The following antibiotics were covered in this study: AM-Ampicillin, AMC-Amoxicillin/Clavulanic Acid, ANAmikacin, ATM-Aztreonam, CAZ-Ceftazidime, CIP-Ciprofloxacin, CM-Clindamycin, CRO-Ceftriaxone, CTX-Cefotaxime, CZCefazolin, E-Erythromycin, ETP-Ertapenem, FA-Fusidic acid, FEP-Cefepime, FOS-Fosfomycin, FT-Nitrofurantoin, GMGentamicin, IPM-Imipenem, LEV-Levofloxacin, LNZ-Linezolid, MEM-Meropenem, MNO-Minocycline, MUP-Mupirocin, MXFMoxifloxacin, OX1-Oxacillin, P-Benzylpenicillin, PEF-Pefloxacin, PIP-Piperacillin, RA-Rifampicin, SAM-Ampicillin/Sulbactam, SXT-Trimethoprim/Sulfamethoxazole, TEC-Teicoplanin, TETetracycline, TGC-Tigecycline, TIC-Ticarcillin, TM-Tobramycin, TZP-Piperacillin/Tazobactam, and VA-Vancomycin.

\section{Data Analysis}

Abundance, distribution, and drug sensitivity of all bacterial isolates were presented in percentile (\%). When the percentile of sensitivity of a certain isolate was $<30 \%$, that isolate was considered to be resistant. For the collective antibiotic resistance in GPB and GNB, drugs were presented in a descending manner and only with a value $\geq 90 \%$ were considered to be highly resistant. For these calculations Microsoft Excel 2010 was used.

\section{RESULTS}

\section{A. Pathogenic Bacteria were Isolated in One Fourth of the Collected Samples}

Pathogenic bacteria were recovered in 71 of the 269 wound samples grown, accounting for $26.39 \%$ of the total samples. Non-pathogenic bacteria were found in $124(46.1 \%)$ of the samples. Meanwhile, no bacterial growth was found in $74(27.51 \%)$ of the samples, (Table 1$)$.

\section{B. Gram-Negative Bacterial is the Dominant Type of Bacteria in Wounds}

In wound isolates from 71 human individuals, pathogenic GNB were shown to be more prevalent than pathogenic GNB. GNB accounted for $42(59.15 \%)$ of the total, while pathogenic GNB accounted for 29 (40.85\%) of total isolates, (Table 2). 
C. E. coli and P. aeruginosa are the Dominant Pathogenic GNB in Wounds

$E$. coli and $P$. aeruginosa showed the higher growth in isolated bacteria representing $13(30.95 \%)$ and $9(21.43 \%)$ of total GNB identified, respectively, followed by Klebsiella pneumonia 5 (11.9\%), Enterobacter cloacae 3 (7.14\%), and Proteus mirabilis 3 (7.14\%). Citrobacter koseri is the bacteria with the least dominance, accounting for only $1(2.38 \%)$ of the total, (Table 3).

\section{S. aureus is the Dominant Pathogenic GPB in Wounds}

More than half of the isolated pathogenic GPB were $S$. aureus, followed by Staphylococcus epidermidis, which constituted one fourth of the total isolates. Staphylococcus haemolyticus and E. faecalis were found in only two colony isolates, as Streptococcus pneumoniae was the rarest. It was found only in one colony isolate, (Table 3).

\section{E. Gram-Negative P. aeruginosa is $100 \%$ Resistant to Most} of the Tested Antibiotics

E. coli showed $100 \%$ resistance to Pefloxacin and Piperacillin. As well as $92.31 \%$ resistance to Monocyline, $84.62 \%$ resistance to Ampicillin, Amoxicillin/Clavulanic Acid, Aztreonam, and Ampicillin/Sulbactam, (Table 4).

TABLE I

Distribution of Bacterial Growth of Wound Swab Culture

\begin{tabular}{lc}
\hline \hline Bacterial growth & Number of growth (\%) \\
\hline No growth of bacteria & $74(27.51 \%)$ \\
No bacterial pathogen isolates & $124(46.1 \%)$ \\
Pathogenic bacteria isolates & $71(26.39 \%)$ \\
\hline \hline
\end{tabular}

TABLE II

Distribution of Pathogenic Bacteria Isolated from Wound Swab Culture

\begin{tabular}{lc}
\hline \hline Bacterial growth & Number of growth (\%) \\
\hline GPB isolates & $29(40.85 \%)$ \\
GNB isolates & $42(59.15 \%)$ \\
\hline \hline
\end{tabular}

TABLE III

Distribution of GNB and GPB Isolated from Wound Swab Culture

\begin{tabular}{|c|c|c|c|}
\hline GNB isolates & $\begin{array}{l}\text { Number of } \\
\text { isolates (\%) }\end{array}$ & GPB isolates & $\begin{array}{c}\text { Number of isolates } \\
(\%)\end{array}$ \\
\hline Escherichia coli & $13(30.95 \%)$ & $\begin{array}{l}\text { Staphylococcus } \\
\text { aureus }\end{array}$ & $17(58.62 \%)$ \\
\hline Pseudomonas aeruginosa & $9(21.43 \%)$ & $\begin{array}{l}\text { Staphylococcus } \\
\text { epidermidis }\end{array}$ & $7(24.13 \%)$ \\
\hline Klebsiella pneumonia & $5(11.9 \%)$ & $\begin{array}{l}\text { Staphylococcus } \\
\text { haemolyticus }\end{array}$ & $2(6.9 \%)$ \\
\hline Enterobacter cloacae & $3(7.14 \%)$ & $\begin{array}{l}\text { Enterococcus } \\
\text { faecalis }\end{array}$ & $2(6.9 \%)$ \\
\hline Proteus mirabilis & $3(7.14 \%)$ & $\begin{array}{l}\text { Streptococcus } \\
\text { pneumoniae }\end{array}$ & $1(3.45 \%)$ \\
\hline Acinetobacter baumannii & $2(4.76 \%)$ & - & - \\
\hline Enterobacter aerogenes & $2(4.76 \%)$ & - & - \\
\hline Klebsiella oxytoca & $2(4.76 \%)$ & - & - \\
\hline Morganella morganii & $2(4.76 \%)$ & - & - \\
\hline Citrobacter koseri & $1(2.38 \%)$ & - & - \\
\hline
\end{tabular}

Whereas, the second dominant Gram-negative pathogen is $P$. aeruginosa showed $100 \%$ resistance to the majority of antibiotics tested, including Ampicillin, Amoxicillin/ Clavulanic Acid, Aztreona, Ceftriaxone, Cefazolin, Ertapenem, Nitrofurantoin, Minocycline, Pefloxacin, Piperacillin, Ampicillin/Sulbactam, Tigecycline, Ticarcillin, and Piperacillin/Tazobactam, (Table 5).

\section{F. The Wound Dominant GPB are $100 \%$ resistant to Ampicillin, Ceftriaxone, and Cefotaxime}

Antibiotic sensitivity test showed that S. aureus is $100 \%$ resistant to Ampicillin, Ceftriaxone, and Cefotaxime, and $64.71 \%$ resistant to Ciprofloxacin. S. epidermidis is $100 \%$ resistant to Ampicillin, Ceftriaxone, and Cefotaxime. It also shows $85 \%$ resistance to Ciprofloxacin, Fusidic acid, and Quinupristin/Dalfopristin. Moreover, S. epidermidis is $71.43 \%$ resistant to Erythromycin, and Teicoplanin, (Table 6).

\section{G. Both GPB and GNB in Wounds are Resistant to Ampicillin}

Collectively GPB were resistant to Ampicillin, Ceftriaxone, and Cefotaxime by $96.55 \%$, followed by Ciprofloxacin and Quinupristin/Dalfopristin by $72.41 \%$ and $68.97 \%$, respectively. Whereas, the bacteria were least resistant to Linezolid by $3.45 \%$, and Fosfomycin, Moxifloxacin, Tigecycline, Benzylpenicillin by $6.89 \%$. Collectively, GNB were resistant to Ampicillin by $92.86 \%$ and Minocycline by $90.48 \%$. Whereas, the least resistance was seen when using Amikacin by $33.33 \%$ and Cefepime by 40.48 , (Table 7).

\section{Discussion}

The over usage of antibiotics is a crucial factor in the development of resistance toward these drugs. Knowing the signs and symptoms of wound infections, as well as the causative organisms, and their current susceptibility trends are essential for pragmatic antibiotic prescribing (Filius and Gyssens, 2002). This study aimed to identify types of bacteria in wounds and their antibiotic susceptibility. Our results indicate that the dominant types of pathogenic bacteria in wounds are GNB E. coli and P. aeruginosa, accounting for $30.95 \%$ and $21.43 \%$ of total GNB, respectively. In wounds, K. pneumoniae $11.9 \%$, E. cloacae $7.14 \%$, and P. mirabilis $7.14 \%$ were found. The GNB with the least amount of dominance is C. koseri with only $2.38 \%$.

Our result is similar to the finding of other research regarding predominance of GNB in wounds. Park, et al. (2017) reported that the majority (52\%) of wound associated pathogens are of GNB. However, the commonalty of the species might show some differences for example in a research by Azzopardi, et al. (2014) found that the most prevalent Gram-negative-burn wound-pathogens were P. aeruginosa, K. pneumoniae, Acinetobacter baumannii, Enterobacter spp., Proteus spp., and E. coli (Azzopardi, et al., 2014; Azzopardi, et al., 2011; Park, et al., 2017).

$S$. aureus accounted for more than half of the pathogenic GPB identified, followed by $S$. epidermidis, which accounted 
TABLE IV

Antimicrobial Agent's Responses to the Isolated GNB

\begin{tabular}{|c|c|c|c|c|c|c|c|}
\hline \multirow{3}{*}{$\begin{array}{l}\text { Agent } \\
\text { AM-Ampicillin } \\
\text { AMC-Amoxicillin/ } \\
\text { Clavulanic Acid }\end{array}$} & \multirow{3}{*}{$\begin{array}{c}\begin{array}{c}\text { Acinetobacter } \\
\text { baumannii }(2)\end{array} \\
\text { R2 }(100 \%) \\
\text { R2 }(100 \%)\end{array}$} & \multirow{3}{*}{$\begin{array}{l}\text { Citrobacter koseri (1) } \\
\text { R1 }(100 \%) \\
\text { R1 }(100 \%)\end{array}$} & \multirow{3}{*}{$\begin{array}{c}\text { Enterobacter aerogenes (2) } \\
\text { R2 }(100 \%) \\
\text { R2 }(100 \%)\end{array}$} & \multicolumn{2}{|c|}{ Enterobacter cloacae (3) } & \multicolumn{2}{|c|}{ Escherichia coli (13) } \\
\hline & & & & \multicolumn{2}{|c|}{ R3 (100\%) } & S2 (15.38\%) & R11 (84.62\%) \\
\hline & & & & S2 (66.67\%) & R1 (33.33\%) & S2 (15.38\%) & R11 $(84.62 \%)$ \\
\hline AN-Amikacin & R2 (100\%) & S1 $(100 \%)$ & S2 $(100 \%)$ & S2 (66.67\%) & R1 (33.33\%) & S7 (53.85\%) & R6 (46.15\%) \\
\hline ATM-Aztreonam & R2 (100\%) & S1 $(100 \%)$ & S2 $(100 \%)$ & S1 $(33.33 \%)$ & R2 (66.67\%) & S2 (15.38\%) & R11 $(84.62 \%)$ \\
\hline CAZ-Ceftazidime & R2 (100\%) & S1 $(100 \%)$ & S2 $(100 \%)$ & S2 (66.67\%) & R1 (33.33\%) & S5 (38.46\%) & R8 (61.54\%) \\
\hline CZ-Cefazolin & R2 (100\%) & S1 $(100 \%)$ & R2 (100\%) & \multicolumn{2}{|c|}{ R3 (100\%) } & S3 (23.08\%) & R10 $(76.92 \%)$ \\
\hline ETP-Ertapenem & R2 (100\%) & S1 $(100 \%)$ & R2 (100\%) & S2 $(66.67 \%)$ & R1 (33.33\%) & S9 $(69.23 \%)$ & R4 (30.77\%) \\
\hline FEP-Cefepime & R2 (100\%) & S1 $(100 \%)$ & $\mathrm{S} 2(100 \%)$ & \multicolumn{2}{|c|}{ S3 $(100 \%)$} & S5 (38.46\%) & R8 (61.54\%) \\
\hline FT-Nitrofurantoin & R2 (100\%) & R1 (100\%) & R2 (100\%) & S1 (33.33\%) & R2 (66.67\%) & S5 (38.46\%) & R8 (61.54\%) \\
\hline GM-Gentamicin & R2 (100\%) & $\mathrm{S} 1(100 \%)$ & $\mathrm{S} 2(100 \%)$ & S1 $(33.33 \%)$ & R2 (66.67\%) & S9 $(69.23 \%)$ & R4 (30.77\%) \\
\hline PEF-Pefloxacin & R2 (100\%) & R1 (100\%) & $\mathrm{S} 2(100 \%)$ & S1 $(33.33 \%)$ & R2 (66.67\%) & \multicolumn{2}{|c|}{ R13 (100\%) } \\
\hline PIP-Piperacillin & R2 (100\%) & R1 (100\%) & $\mathrm{S} 2(100 \%)$ & \multicolumn{2}{|c|}{ R3 (100\%) } & $\mathrm{R} 13$ & $00 \%)$ \\
\hline $\begin{array}{l}\text { SAM-Ampicillin/ } \\
\text { Sulbactam }\end{array}$ & S2 (100\%) & R1 (100\%) & R2 (100\%) & \multicolumn{2}{|c|}{ R3 (100\%) } & S2 (15.38\%) & R11 $(84.62 \%)$ \\
\hline $\begin{array}{l}\text { SXT-Trimethoprim/ } \\
\text { Sulfamethoxazole }\end{array}$ & S2 (100\%) & S1 (100\%) & S2 (100\%) & S1 $(33.33 \%)$ & R2 (66.67\%) & S7 (53.85\%) & R6 (46.15\%) \\
\hline TGC-Tigecycline & R2 (100\%) & S1 $(100 \%)$ & R2 (100\%) & S1 $(33.33 \%)$ & R2 (66.67\%) & S4 (30.77\%) & R9 (69.23\%) \\
\hline TIC-Ticarcillin & R2 (100\%) & R1 (100\%) & $\mathrm{S} 2(100 \%)$ & \multicolumn{2}{|c|}{ R3 $(100 \%)$} & \multicolumn{2}{|c|}{ R13 (100\%) } \\
\hline TM-Tobramycin & $\mathrm{S} 1(50 \%)$ & $\mathrm{R} 2(50 \%) \quad \mathrm{S} 1(100 \%)$ & S2 $(100 \%)$ & \multicolumn{2}{|c|}{$\begin{array}{l}\text { R3 }(100 \%) \\
\text { S3 }(100 \%)\end{array}$} & S6 (46.15\%) & R7 (53.85\%) \\
\hline $\begin{array}{l}\text { TZP-Piperacillin/ } \\
\text { Tazobactam }\end{array}$ & R2 (100\%) & S1 $(100 \%)$ & S2 (100\%) & & & S6 (46.15\%) & R7 (53.85\%) \\
\hline
\end{tabular}

S: Sensitive, R: Resistant

TABLE V

Antimicrobial Agent's Responses To The Isolated GNB

\begin{tabular}{|c|c|c|c|c|c|}
\hline Agent & Klebsiella oxytoca $(2)$ & $\begin{array}{c}\text { Klebsiella } \\
\text { pneumonia }(5)\end{array}$ & $\begin{array}{l}\text { Morganella } \\
\text { morganii (2) }\end{array}$ & Proteus mirabilis (3) & $\begin{array}{c}\text { Pseudomonas aeruginosa } \\
\text { (9) }\end{array}$ \\
\hline AM-Ampicillin & R2 $(100 \%)$ & R5 $(100 \%)$ & R2 (100\%) & $\mathrm{S} 1(33.33 \%)$ R2 (66.67\%) & R9 $(100 \%)$ \\
\hline AMC-Amoxicillin/Clavulanic Acid & R2 (100\%) & S1 (20\%) R4 (80\%) & R2 $(100 \%)$ & $\mathrm{S} 1(33.33 \%)$ R2 (66.67\%) & R9 (100\%) \\
\hline ATM-Aztreonam & $\mathrm{S} 2(100 \%)$ & R5 (100\%) & S2 $(100 \%)$ & $\mathrm{S} 1(33.33 \%)$ R2 (66.67\%) & R9 (100\%) \\
\hline CAZ-Ceftazidime & $\mathrm{S} 2(100 \%)$ & R5 (100\%) & S2 $(100 \%)$ & S2 (66.67\%) R1 (33.33\%) & S5 (55.56\%) R4 (44.44\%) \\
\hline CIP-Ciprofloxacin & $\mathrm{S} 2(100 \%)$ & S1 $(20 \%)$ R4 (80\%) & S2 $(100 \%)$ & S2 (66.67\%) R1 (33.33\%) & S4 (44.44\%) R5 (55.56\%) \\
\hline ETP-Ertapenem & $\mathrm{S} 2(100 \%)$ & $\mathrm{S} 3(60 \%)$ R2 (40\%) & R2 (100\%) & $\mathrm{S} 1(33.33 \%)$ R2 (66.67\%) & R9 (100\%) \\
\hline FEP-Cefepime & $\mathrm{S} 2(100 \%)$ & R5 (100\%) & S2 $(100 \%)$ & S2 (66.67\%) R1 (33.33\%) & S8 (88.89\%) R1 (11.11\%) \\
\hline FT-Nitrofurantoin & $\mathrm{R} 2(100 \%)$ & S1 (20\%) R4 (80\%) & $\mathrm{R} 2(100 \%)$ & R3 (100\%) & R9 (100\%) \\
\hline GM-Gentamicin & $\mathrm{S} 2(100 \%)$ & S1 $(20 \%)$ R4 (80\%) & $\mathrm{S}(50 \%) \quad \mathrm{R} 1(50 \%)$ & S1 (33.33\%) R2 (66.67\%) & S6 (66.67\%) R3 (33.33\% \\
\hline IPM-Imipenem & $\mathrm{S} 2(100 \%)$ & $\mathrm{S} 3(60 \%) \mathrm{R} 2(40 \%)$ & $\mathrm{R} 2(100 \%)$ & R3 (100\%) & S8 (88.89\%) R1 (11.11\%) \\
\hline PIP-Piperacillin & R2 (100\%) & R5 (100\%) & S2 $(100 \%)$ & $\mathrm{S} 1(33.33 \%)$ R2 (66.67\%) & R9 (100\%) \\
\hline SAM-Ampicillin/Sulbactam & $\mathrm{S} 2(100 \%)$ & R5 (100\%) & $\mathrm{R} 2(100 \%)$ & $\mathrm{S} 1(33.33 \%) \mathrm{R} 2(66.67 \%)$ & R9 (100\%) \\
\hline SXT-Trimethoprim/Sulfamethoxazole & $\mathrm{S} 2(100 \%)$ & S1 (20\%) R4 (80\%) & $\mathrm{S} 1(50 \%) \mathrm{R} 1(50 \%)$ & R3 (100\%) & S6 (66.67\%) R3 (33.33\% \\
\hline TGC-Tigecycline & S2 $(100 \%)$ & S2 (40\%) R3 (60\%) & $\mathrm{R} 2(100 \%)$ & R3 (100\%) & R9 (100\%) \\
\hline TIC-Ticarcillin & S2 $(100 \%)$ & R5 (100\%) & S2 $(100 \%)$ & S1 $(33.33 \%)$ R2 (66.67\%) & R9 (100\%) \\
\hline TM-Tobramycin & S2 $(100 \%)$ & R5 (100\%) & S2 $(100 \%)$ & S2 (66.67\%) R1 (33.33\%) & S8 (88.89\%) R1 (11.11\%) \\
\hline TZP-Piperacillin/Tazobactam & S2 $(100 \%)$ & R5 (100\%) & S2 $(100 \%)$ & S2 (66.67\%) R1 (33.33\%) & R9 $(100 \%)$ \\
\hline
\end{tabular}

S: Sensitive, R: Resistant 
TABLE VI

Antimicrobial Agent's Responses To The Isolated GPB

\begin{tabular}{|c|c|c|c|c|c|}
\hline Agent & $\begin{array}{l}\text { Enterococcus } \\
\text { faecalis (2) }\end{array}$ & Staphylococcus aureus (17) & $\begin{array}{c}\text { Staphylococcus epidermidis } \\
\text { (7) }\end{array}$ & $\begin{array}{l}\text { Staphylococcus } \\
\text { haemolyticus (2) }\end{array}$ & $\begin{array}{c}\text { Streptococcus } \\
\text { pneumoniae }(1)\end{array}$ \\
\hline AM-Ampicillin & R2 (100\%) & R17 (100\%) & R7 (100\%) & R2 (100\%) & S1 $(100 \%)$ \\
\hline CIP-Ciprofloxacin & $\mathrm{R} 2(100 \%)$ & S6 (35.29\%) R11 (64.71\%) & S1 (14.29\%) R6 (85.71\%) & R2 (100\%) & S1 $(100 \%)$ \\
\hline CRO-Ceftriaxone & $\mathrm{R} 2(100 \%)$ & R17 (100\%) & R7 (100\%) & R2 (100\%) & S1 $(100 \%)$ \\
\hline CTX-Cefotaxime & R2 (100\%) & R17 (100\%) & R7 (100\%) & R2 (100\%) & $\mathrm{S} 1(100 \%)$ \\
\hline E-Erythromycin & $\mathrm{R} 2(100 \%)$ & $\mathrm{S} 12(70.59 \%) \quad \mathrm{R} 5(29.41 \%)$ & S2 (28.57\%) R5 (71.43\%) & R2 (100\%) & S1 $(100 \%)$ \\
\hline FT-Nitrofurantoin & $\mathrm{S} 2(100 \%)$ & S17 (100\%) & S7 (100\%) & $\mathrm{S} 2(100 \%)$ & $\mathrm{S} 1(100 \%)$ \\
\hline GM-Gentamicin & R2 (100\%) & $\mathrm{S} 16(94.12 \%) \quad \mathrm{R} 1(5.88 \%)$ & S7 (100\%) & $\mathrm{S} 1(50 \%) \mathrm{R} 1(50 \%)$ & $\mathrm{S} 1(100 \%)$ \\
\hline LEV-Levofloxacin & R2 $(100 \%)$ & $\mathrm{S} 16(94.12 \%) \quad \mathrm{R} 1(5.88 \%)$ & S7 (100\%) & $\mathrm{S} 1(50 \%) \mathrm{R} 1(50 \%)$ & S1 $(100 \%)$ \\
\hline LNZ-Linezolid & $\mathrm{S} 1(50 \%) \mathrm{R} 1(50 \%)$ & S17 (100\%) & S7 (100\%) & $\mathrm{S} 2(100 \%)$ & $\mathrm{S} 1(100 \%)$ \\
\hline MUP-Mupirocin & R2 (100\%) & S9 (52.94\%) R8 (47.06\%) & S5 (71.43\%) R2 (28.57\%) & R2 (100\%) & R1 (100\%) \\
\hline RA-Rifampicin & $\mathrm{R} 2(100 \%)$ & $\mathrm{S} 16(94.12 \%) \quad \mathrm{R} 1(5.88 \%)$ & S3 (42.86\%) R4 (157.14\%) & $\mathrm{R} 2(100 \%)$ & R1 (100\%) \\
\hline SXT-Trimethoprim/Sulfamethoxazole & R2 (100\%) & $\mathrm{S} 16(94.12 \%) \quad \mathrm{R} 1(5.88 \%)$ & S5 (71.43\%) R2 (28.57\%) & $\mathrm{S} 2(100 \%)$ & $\mathrm{R} 1(100 \%)$ \\
\hline TE-Tetracycline & $\mathrm{R} 2(100 \%)$ & S9 (52.94\%) R8 (47.06\%) & S3 (42.86\%) R4 (157.14\%) & $\mathrm{S} 1(50 \%) \mathrm{R} 1(50 \%)$ & $\mathrm{S} 1(100 \%)$ \\
\hline TEC-Teicoplanin & $\mathrm{S} 2(100 \%)$ & $\mathrm{S} 10(58.82 \%) \quad \mathrm{R} 7(41.18 \%)$ & S2 (28.57\%) R5 (71.43\%) & R2 (100\%) & R1 $(100 \%)$ \\
\hline TGC-Tigecycline & S2 $(100 \%)$ & $\mathrm{S} 15(88.24 \%) \quad \mathrm{R} 2(11.76 \%)$ & S7 $(100 \%)$ & S2 $(100 \%)$ & $\mathrm{S} 1(100 \%)$ \\
\hline TM-Tobramycin & R2 (100\%) & S9 (52.94\%) R8 (47.06\%) & S6 (85.71\%) R1 (14.29\%) & $\mathrm{S} 1(50 \%) \mathrm{R} 1(50 \%)$ & R1 (100\%) \\
\hline VA-Vancomycin & $\mathrm{S} 2(100 \%)$ & $\mathrm{S} 15(88.24 \%) \quad \mathrm{R} 2(11.76 \%)$ & S3 (42.86\%) R4 (57.14\%) & $\mathrm{S} 1(50 \%) \mathrm{R} 1(50 \%)$ & $\mathrm{S} 1(100 \%)$ \\
\hline
\end{tabular}

S: Sensitive, R: Resistant

TABLE VII

Pattern Of Antimicrobial Resistance Among Detected Bacteria

\begin{tabular}{|c|c|c|c|c|}
\hline \multirow[t]{2}{*}{ S. No. } & \multicolumn{2}{|l|}{ GPB } & \multicolumn{2}{|l|}{ GNB } \\
\hline & Antibiotics & $\%$ Resistance & Antibiotics & $\%$ Resistance \\
\hline 1. & AM-Ampicillin & 96.55 & AM-Ampicillin & 92.86 \\
\hline 2. & CRO-Ceftriaxone & 96.55 & MNO-Minocycline & 90.48 \\
\hline 3. & CTX-Cefotaxime & 96.55 & PIP-Piperacillin & 88.1 \\
\hline 4. & CIP-Ciprofloxacin & 72.41 & AMC-Amoxicillin/Clavulanic Acid & 85.71 \\
\hline 5. & QDA-Quinupristin/Dalfopristin & 68.97 & CZ-Cefazolin & 83.33 \\
\hline 8. & TE-Tetracycline & 51.72 & SAM-Ampicillin/Sulbactam & 83.33 \\
\hline 9. & TEC-Teicoplanin & 51.72 & TIC-Ticarcillin & 83.33 \\
\hline 10 & E-Erythromycin & 48.28 & CRO-Ceftriaxone & 80.95 \\
\hline 11. & CM-Clindamycin & 44.83 & TGC-Tigecycline & 76.19 \\
\hline 12. & TM-Tobramycin & 44.83 & TZP-Piperacillin/Tazobactam & 71.43 \\
\hline 13. & OX1-Oxacillin & 34.48 & LEV-Levofloxacin & 69.05 \\
\hline 18. & LEV-Levofloxacin & 13.79 & SXT-Trimethoprim/Sulfamethoxazole & 45.24 \\
\hline 19. & FOS-Fosfomycin & 6.89 & TM-Tobramycin & 45.24 \\
\hline 20. & MXF-Moxifloxacin & 6.89 & GM-Gentamicin & 42.86 \\
\hline 21. & P-Benzylpenicillin & 6.89 & FEP-Cefepime & 40.48 \\
\hline 22. & TGC-Tigecycline & 6.89 & MEM-Meropenem & 38.1 \\
\hline 23. & LNZ-Linezolid & 3.45 & AN-Amikacin & 33.33 \\
\hline 24. & - & - & IPM-Imipenem & 26.19 \\
\hline
\end{tabular}

for one-fourth of the total isolates. Only two colony isolates contained S. haemolyticus and E. faecalis, with S. pneumoniae being the least common. This finding is supported by other studies since $S$. aureus is reported to be the common GPB in wound patients (Mahat, et al., 2017; Guan, et al., 2021).

There seems to be variability in the diversity of bacterial culture in wounds. A study done by Bessa, et al. (2015) showed that the most common bacterial species detected was 
S. aureus (37\%), followed by P. aeruginosa (17\%), P. mirabilis (10\%), E. coli (6\%), and Corynebacterium spp. Our results came in agreement with theirs regarding antibiotic sensitivity tests as they also reported that Vancomycin and Linezolid were effective against all GPB, and GNB revealed a significant level of resistance to the majority of antibiotics with Amikacin being the most effective against them (Bessa, et al., 2015).

In general, speaking, GPB were highly resistant toward each of Ampicillin, Ceftriaxone, and Cefotaxime. Meanwhile, they were highly sensitive to Fosfomycin, Moxifloxacin, P-Benzylpenicillin, Tigecycline, and Linezolid specifically. For being resistant by $100 \%$ to 19 (out of 24) drugs, E. faecalis was the most resistant recovered GPB. Since S. pneumoniae was sensitive by $100 \%$ toward 16 (out of 24) drugs it was the most sensitive recovered GPB (Tables 6 and 7).

These results indicate that multidrug-resistant (MDR) bacteria were associated with the identified pathogens and may play a negative role in chronic wound infection. These findings come in agreement with previous studies of ours that confirmed the existence of MDR bacteria among patients identified with urinary tract and lower respiratory tract infections (Al-Naqshbandi, et al., 2019; Chawsheen, et al., 2020; Abbas and Owaid, 2021).

\section{CONCLUSION}

It is concluded from this research the importance of learning more about the causes of wound infections and the criteria for detecting them so that more appropriate antibiotics may be prescribed. GNB made up to two third of total bacterial isolates cultured from wounds. The most dominant pathogenic GNB were E. coli and P. aeruginosa. The most dominant pathogenic GNB isolated from wounds were $S$. aureus and $S$. epidermidis. The majority of antibiotics tested, including ampicillin, amoxicillin/clavulanic acid, Aztreona, Ceftriaxone, and others, exhibited 100\% resistance. Ampicillin, Ceftriaxone, and Cefotaxime are completely ineffective against $S$. aureus and $S$. epidermidis. The pathogenic bacteria and their current susceptibility patterns must be enforced for testing before administering any antibiotics to patients for more efficient antibiotic prescriptions.

\section{RECOMMENDATIONS}

A significant number of MDR bacteria were recognized as the causal agents of wound infection in this study. Mandatory investigation of wound specimens, as well as antibiotic susceptibility testing, is recommended to guide physicians in pragmatic wound infection therapy, thereby reducing the propagation of resistant bacteria.

\section{Author CONTRIBUtions}

The $1^{\text {st }}$ and $4^{\text {th }}$ authors contributed to this study by collecting data and conducting laboratory techniques. The $2^{\text {nd }}$ and $3^{\text {rd }}$ authors contributed in writing this manuscript. Data analysis was conducted by the $1^{\text {st }}$ and $3^{\text {rd }}$ authors.

\section{ACKNOWLEDGMENTS}

We would like to acknowledge the instrumental support of Rizgary Teaching Hospital to complete this study.

\section{CONFLICT OF INTEREST}

The authors declare no conflict of interest.

\section{REFERENCES}

Abbas, Z.K. and Owaid, M.N., 2021. Prevalence of antibiotics resistance in the isolated bacteria from bronchial washing fluids in Ramadi Teaching Hospital, Iraq. Gazi Medical Journal, 32(3), pp.359-363.

Al-Naqshbandi, A.A., Chawsheen, M.A. and Abdulqader, H.H., 2019. Prevalence and antimicrobial susceptibility of bacterial pathogens isolated from urine specimens received in Rizgary hospital-Erbil. Journal of Infection and Public Health, 12, pp.330-336.

Azzopardi, E.A., Azzopardi, S.M., Boyce, D.E. and Dickson, W.A., 2011. Emerging gram-negative infections in burn wounds. Journal of Burn Care and Research, 32, pp.570-576.

Bessa, L.J., Fazii, P., Di Giulio, M. and Cellini, L., 2015. Bacterial isolates from infected wounds and their antibiotic susceptibility pattern: Some remarks about wound infection. International Wound Journal, 12, 47-52.

Bjarnsholt, T., 2013. The role of bacterial biofilms in chronic infections. Apmis, 121, pp.1-58

Bowler, P.G., 2018. Antibiotic resistance and biofilm tolerance: A combined threat in the treatment of chronic infections. Journal of Wound Care, 27, pp.273-277.

Chadwick, P. and Ousey, K., 2019. Bacterial-binding dressings in the management of wound healing and infection prevention: A narrative review. Journal of Wound Care, 28, pp.370-382.

Chawsheen, M.A., Al-Naqshbandi, A.A. and Abdulqader, H.H., 2020. Bacterial profile and antimicrobial susceptibility of isolates recovered from lower respiratory tract infection for patients in Rizgary Hospital, Erbil. Aro-the Scientific Journal of Koya University, 8, pp.64-70.

Cooper, R.A., Bjarnsholt, T. and Alhede, M., 2014. Biofilms in wounds: A review of present knowledge. Journal of Wound Care, 23, pp.570-582.

Daeschlein, G., 2013. Antimicrobial and antiseptic strategies in wound management. International Wound Journal, 10, pp.9-14.

Demidova-Rice, T.N., Hamblin, M.R. and Herman, I.M., 2012. Acute and impaired wound healing. Advances in Skin and Wound Care, 25, pp.304-314.

Filius, P.M. and Gyssens, I.C., 2002. Impact of increasing antimicrobial resistance on wound management. American Journal of Clinical Dermatology, 3, pp.1-7.

Frykberg, R.G. and Banks, J., 2015. Challenges in the treatment of chronic wounds. Advances in Wound Care, 4, pp.560-582.

Guan, H., Dong, W., Lu, Y., Jiang, M., Zhang, D., Aobuliaximu, Y., Dong, J., Niu, Y., Liu, Y., Guan, B., Tang, J. and Lu, S., 2021. Distribution and antibiotic resistance patterns of pathogenic bacteria in patients with chronic cutaneous wounds in China. Frontiers in Medicine, 8, pp.609584.

Herman, T.F. and Bordoni, B., 2021. Wound classification. In: StatPearls. StatPearls Publishing, Treasure Island, FL.

Kumar, S., 2016. Essentials of Microbiology. Jaypee Group, Noida. 
Mcdowell, A., Azzopardi, E.A., Azzopardi, E., Camilleri, L., Villapalos, J., Boyce, D.E., Dziewulski, P., Dickson, W.A. and Whitaker, I.S., 2014. Gram negative wound infection in hospitalised adult burn patients-systematic review and metanalysis. PLoS One, 9, p.e95042.

Negut, I., Grumezescu, V. and Grumezescu, A., 2018. Treatment strategies for infected wounds. Molecules, 23, p.2392.

Park, H.S., Pham, C., Paul, E., Padiglione, A., Lo, C. and Cleland, H., 2017. Early pathogenic colonisers of acute burn wounds: A retrospective review. Burns, 43, pp.1757-1765.
Pushpa, M., 2017. Bacteriological profile of wound infection and antibiotic susceptibility pattern of the isolates. Journal of Microbiology and Experimentation, 4, pp.119-122.

Reale, M., Strazzulla, A., Quirino, A., Rizzo, C., Marano, V., Postorino, M.C., Mazzitelli, M., Greco, G., Pisani, V., Costa, C., Cesana, B.M., Liberto, M.C., Torti, C. and Foca, A., 2017. Patterns of multi-drug resistant bacteria at first culture from patients admitted to a third level University hospital in Calabria from 2011 to 2014: Implications for empirical therapy and infection control. Infezioni in Medicina, 25, pp.98-107. 